\title{
Perfil epidemiológico dos pacientes com ptose congênita no hospital regional de São José
}

\author{
Epidemiological profile of congenital ptosis \\ pacients in the hospital regional de São José
}

Astor Grumann Júnior' ${ }^{1}$ Felipe Roberto Exterhotter Branco²

\section{Resumo}

Objetivo: Traçar um perfil epidemiológico dos pacientes com ptose congênita no Hospital Regional de São José (HRSJ), descrevendo as características gerais das ptoses congênitas, incluindo a coexistência de estrabismo e a prevalência de ambliopia. Métodos: Foi realizado um estudo epidemiológico com delineamento transversal, baseado na análise dos prontuários de pacientes com ptose congênita atendidos no Departamento de Plástica Ocular e Órbita do HRSJ, no período de julho de 1998 a julho de 2008. Resultados: Foram analisados 42 pacientes (56 olhos). A idade média foi de 7,2 anos e o gênero mais prevalente foi o masculino (66,7\%). Foi encontrado unilateralidade da ptose em 66,7\% dos casos, associação com estrabismo em $19 \%$ e fenômeno de Marcus Gunn em 9,5\%. Ambliopia foi encontrada em $17 \%$ dos olhos afetados. Na classificação, 38,5\% dos olhos tinham ptose severa e $63 \%$ tinham excursão do elevador fraca ou ausente. A conduta foi cirúrgica para 57,2\% dos casos e a técnica mais prevalente foi elevação ao Frontal (75\%). Conclusão: No presente estudo, a prevalência de ambliopia nos pacientes com ptose congênita foi maior que da população geral, reforçando a importância de uma avaliação oftalmológica precoce destes pacientes.

Descritores: Doenças palpebrais; Blefaroptose/congênito; Prevalência; Ambliopia

\begin{abstract}
Purpose: To draw an epidemiological profile of congenital ptosis patients in the Hospital Regional de São José (HRSJ), describing the general characteristics of congenital ptosis, including the coexistence of strabismus and the prevalence of amblyopia. Methods: An epidemiological study with a cross-sectional characteristic was conducted, based on analysis of medical records of congenital ptosis cases treated on the Department of Ocular Plastic and Orbit of the HRSJ, from July 1998 to July 2008. Results: A total of 42 patients (56 eyes) were analyzed. The mean age was 7,2 years and the most prevalent genre was male $(66,7 \%)$. Unilateral ptosis was found in $66,7 \%$ of cases, association with strabismus in $19 \%$ and Marcus Gunn phenomenon in $9,5 \%$. Amblyopia was found in $17 \%$ of the affected eyes. About the classification, $38,5 \%$ of eyes had severe ptosis and $63 \%$ had poor or absent levator excursion. The management for $57,2 \%$ of cases was surgery and the most prevalent technique was Frontal elevation (75\%). Conclusion: In this study, the prevalence of amblyopia in congenital ptosis patients was higher than in the general population, showing the importance of an early ophthalmologic evaluation of these patients.

Keywords: Eyelid diseases; Blepharoptosis/congenital; Prevalence; Amblyopia

\footnotetext{
'Doutor em Oftalmologia, Médico Assistente do Hospital Regional São José - (HRSJ) - São José (SC), Brasil. Professor de Oftalmologia da Universidade do Sul de Santa Catarina - (UNISUL) - Unidade Pedra Branca, (SC), Brasil;

${ }^{2}$ Médico oftalmologista - São José (SC), Brasil.

Trabalho realizado no Departamento de Plástica Ocular e Órbita do Hospital Regional de São José - HRSJ - São José (SC), Brasil.

Os autores declaram inexistir conflitos de interesse

Recebido para publicação em: 27/8/2010 - Aceito para publicação em 30/11/2011
}

Rev Bras Oftalmol. 2011; 70 (6): 391-5 


\section{INTRODUÇÃO}

A pálpebra superior tem papel fundamental na proteção e lubrificação da superfície ocular. Sua elevação é dada principalmente pelo músculo elevador da pálpebra superior (MEPS), inervado pelo terceiro par craniano. Atuam como coadjuvantes nessa função o músculo de Müller e o músculo frontal ${ }^{(1,2)}$.

Ptose ou blefaroptose é o resultado da disfunção isolada ou conjunta dos músculos que atuam na elevação da pálpebra, resultando na queda da margem palpebral para uma posição mais baixa que o normal, na posição primária do olhar ${ }^{(1-3)}$.

Existem diversas classificações para ptoses, sendo que a divisão em congênitas ou adquiridas é a mais utilizada $^{(3,4)}$. A ptose congênita apresenta-se ao nascimento ou dentro do primeiro ano de vida, pode ser unilateral ou bilateral, e não há preferência por sexo ou raça ${ }^{(5)}$. Na maioria das ptoses congênitas verdadeiras o defeito é produzido por distrofia do MEPS, e apresenta-se como uma condição isolada ou associada a outras alterações congênitas como a blefarofimose ${ }^{(3-6)}$. Outros mecanismos, embora menos comuns, também podem causar ptose congênita, como os defeitos neurogênicos que ocorrem na síndrome de Horner congênita e no fenômeno de Marcus Gunn ${ }^{(2,4,6)}$.

As experiências visuais na infância têm grande influência no desenvolvimento das vias visuais, particularmente do córtex visual, que é rudimentar no recémnascido. Com a falta de estimulação dessas vias por obstrução mecânica da pálpebra, não haverá uma maturação adequada do córtex visual no cérebro em desenvolvimento da criança, resultando em ambliopia, ou seja, redução da acuidade visual sem que haja qualquer lesão ocular, que pode variar de uma pequena alteração da função visual a uma perda importante da visão. Contudo, se os fatores de risco ambliogênicos são identificados e tratados cedo, a plasticidade cerebral deverá reorganizar o córtex e a perda visual poderá ser corrigida naturalmente ${ }^{(7-9)}$. Por isso, a medida da acuidade visual e a refração devem ser obtidas na primeira avaliação e a simples suspeita de ambliopia exige que a decisão cirúrgica seja tomada imediatamente ${ }^{(6)}$. A ambliopia também pode levar ao estrabismo pelo pobre desenvolvimento da função ocular ${ }^{(8,9)}$.

O tratamento das ptoses é inteiramente cirúrgi$\mathrm{Co}^{(4)}$. Se a intervenção não for urgente, a cirurgia pode ser prorrogada até a idade de três a quatro anos, quando mensurações mais acuradas poderão ser realizadas no pré-operatório, permitindo um melhor resultado ${ }^{(5)}$.
A avaliação inicial da criança com ptose congênita inclui a determinação da distância margem-reflexo (DMR), que avalia a queda palpebral na posição primária do olhar, medida da excursão do elevador, altura da prega palpebral, e a exclusão de situações sindrômicas como o fenômeno de Marcus Gunn ${ }^{(3)}$. O que se recomenda atualmente é usar a técnica de elevação ao frontal quando houver pobre função do MEPS, o que ocorre na maioria das ptoses congênitas ${ }^{(3,4)}$, e usar qualquer um dos outros métodos dependendo da DMR quando houver boa função do MEPS ${ }^{(4)}$.

Se não corrigidas, as ptoses podem acarretar efeitos psicossociais negativos, perda de autoestima, alienação ${ }^{(10)}$ e desvantagens profissionais ligadas à baixa acuidade visual na vida adulta ${ }^{(8)}$.

Este trabalho foi realizado com o objetivo de traçar um perfil epidemiológico dos pacientes com ptose congênita no Hospital Regional de São José, descrevendo as características gerais das ptoses congênitas, incluindo a coexistência de estrabismo e a prevalência de ambliopia.

\section{Métodos}

Foi realizado um estudo epidemiológico com delineamento transversal, baseado na análise de prontuários dos pacientes com ptose congênita atendidos no Departamento de Plástica Ocular e Órbita do Hospital Regional de São José (HRSJ), no período de julho de 1998 a julho de 2008. Foram incluídas apenas as fichas do primeiro atendimento de cada paciente, não sendo utilizado dados das consultas subsequentes. Foram excluídos todos os casos de ptose com outra classificação que não congênita.

Para definição do ponto de corte foram utilizadas referências encontradas na literatura médica. Sendo assim, foi considerado ambliopia os casos em que a acuidade visual foi menor ou igual a 20/40 ou se houve mais de duas linhas de Snellen de diferença entre os dois olhos. Quanto à excursão do músculo elevador da pálpebra superior, foi considerado "normal" quando esta teve $15 \mathrm{~mm}$ ou mais, "boa" quando teve de 8 a $14 \mathrm{~mm}$, "regular" nos valores de 5 a $7 \mathrm{~mm}$, "fraca" quando menor ou igual a $4 \mathrm{~mm}$ e "ausente" quando nula. Para a classificação da ptose foi utilizada a distância margem-reflexo, onde a queda palpebral foi considerada "mínima" quando o valor encontrado foi de $2 \mathrm{~mm}$, "moderada" quando $1 \mathrm{~mm}$ e "severa" quando $0 \mathrm{~mm}$ ou valores negativos.

Este trabalho foi aprovado pelo do Comitê de Ética em Pesquisa da UNISUL sob o registro número 08.628.4.01.III. 


\section{Resultados}

Em um total de 42 pacientes com ptose congênita, 14 eram do gênero feminino $(33,3 \%)$ e 28 eram do gênero masculino (66,7\%). A média da idade foi de 7,2 anos (mediana de 6 anos), com seus extremos variando de 6 meses a 20 anos.

De acordo com a percepção dos pacientes e/ou familiares, ou por meio de comparação por fotografias antigas, 8 pacientes (19\%) obtiveram algum agravo da ptose ao longo dos anos, 5 (11,9\%) obtiveram alguma melhora e 29 (69\%) tiveram evolução estável desde o nascimento. Apenas 5 pacientes $(11,9 \%)$ tinham história familiar de ptose.

Em relação à lateralidade, 14 pacientes $(33,3 \%)$ apresentaram ptose em ambos os olhos e $28(66,7 \%)$ apresentaram ptose unilateral. Analisando-se somente as ptoses unilaterais, $12(42,9 \%)$ eram no olho direito e $16(57,1 \%)$ no olho esquerdo. No momento da primeira consulta, 13 pacientes $(31 \%)$ haviam tentado algum tipo de tratamento anterior.

A posição da cabeça com o mento elevado ou "chin-up" foi notória em 15 pacientes $(35,7 \%)$, sendo que em 10 destes $(66,6 \%)$ a ptose era bilateral.

Quanto a outras doenças ou fatores associados, 8 pacientes $(19 \%)$ apresentaram estrabismo, 4 (9,5\%) apresentaram o fenômeno de Marcus Gunn, 1 possuía a síndrome da blefarofimose e 1 apresentou glaucoma associado. Não houve história de antecedentes familiares no único caso de blefarofimose encontrado, mas ocorreu em 1 dos 4 casos de fenômeno de Marcus Gun e em 1 dos 8 casos de estrabismo.

Por serem dados que dependem de avaliação por medidas, incluindo o uso de régua milimetrada e cartaz de Snellen, os resultados a seguir estão incompletos na grande maioria das variáveis, principalmente porque não houve colaboração do paciente no momento da consulta para a realização do exame. As percentagens foram dadas em relação aos dados válidos, desconsiderando-se os dados faltantes para o cálculo destas.

Foram analisados 56 olhos ptóticos de 42 pacientes. A média da fenda palpebral de 52 olhos foi de $6 \mathrm{~mm}$ $( \pm 2,06 \mathrm{~mm})$. Outros achados do exame oftalmológico dirigido estão descritos na tabela1.

Apenas para fins descritivos, se for analisado somente as ptoses de classificação severa, verifica-se uma prevalência de fraca excursão do elevador em 71,4\% dos olhos.

As condutas adotadas foram descritas na tabela 2 . A mediana da idade dos pacientes com indicação cirúr-
Tabela 1

Achados do exame oftalmológico dirigido em relação ao número de olhos afetados

\begin{tabular}{lrr}
\hline Exame oftalmológico dirigido & N & \% \\
\hline Acuidade visual & 47 & 100 \\
Normal & 34 & 72,4 \\
Acompanha objetos & 5 & 10,6 \\
Ambliopia & 8 & 17 \\
Classificação da ptose & 39 & 100 \\
Mínima & 11 & 28,2 \\
Moderada & 13 & 33,3 \\
Severa & 15 & 38,5 \\
Excursão do MEPS & 46 & 100 \\
Normal & 2 & 4,4 \\
Boa & 7 & 15,2 \\
Regular & 8 & 17,4 \\
Fraca & 25 & 54,3 \\
Ausente & 4 & 8,7 \\
Força do músculo Frontal & 37 & 100 \\
Normal & 32 & 86,5 \\
Alterada & 5 & 13,5 \\
Prega palpebral & 40 & 100 \\
Presente & 19 & 47,5 \\
Ausente & 21 & 52,5 \\
Reflexo de Bell & 47 & 100 \\
Presente & 36 & 76,6 \\
Ausente ou diminuído & 11 & 23,4 \\
Eixo visual & 38 & 100 \\
Normal & 28 & 73,7 \\
Alterado & 10 & 26,3 \\
\hline
\end{tabular}

(MEPS) músculo elevador da pálpebra superior

Tabela 2

Conduta recomendada após a primeira avaliação oftalmológica dos pacientes com ptose congênita

\begin{tabular}{lrrrr}
\hline Conduta & $\mathbf{N}$ & $\mathbf{\%}$ & $\mathbf{N}$ & $\mathbf{\%}$ \\
\hline Observação & 10 & 23,8 & & \\
Correção cirúrgica & 24 & 57,2 & & \\
Elevação ao frontal & & & 18 & 75 \\
Ressecção do elevador & & & 4 & 16,7 \\
Fassanella-Servat & & & 2 & 8,3 \\
Outras & 8 & 19 & & \\
\hline Total & 42 & 100 & & \\
\hline
\end{tabular}

(DMR) distância margem-reflexo 
gica foi de 7,5 anos, variando de 8 meses a 20 anos, e a mediana da idade dos pacientes sem indicação cirúrgica foi de 5 anos, variando de 6 meses a 19 anos.

\section{DiscuSSÃO}

Iniciando pela análise do gênero, foi notória a relação 2:1 para o gênero masculino, resultado muito próximo ao encontrado em um recente estudo norte-americano, onde $64 \%$ eram do gênero masculino ${ }^{(11)}$. Nas ptoses de etiologias diversas não tem sido encontrado diferença significativa entre os $\operatorname{sexos}^{(12,13)}$.

Quanto à evolução, as ptoses congênitas tendem a ser estáveis, pois sua etiologia está relacionada à distrofia do MEPS, presente desde o nascimento ${ }^{(5,6)}$.Analisando os resultados deste estudo neste ponto, uma explicação para a percepção de melhora do grau de ptose em sua evolução em $11,9 \%$ dos pacientes seria o melhor controle pela criança dos músculos adjuvantes da elevação da pálpebra, o que causaria uma falsa impressão de melhora da ptose. E quanto à percepção de piora, talvez a queda palpebral nos primeiros meses de vida não tenha chamado a atenção dos pais pelas dimensões gerais pequenas do lactente e, mais tarde, com o desenvolvimento dos componentes faciais, a ptose tornou-se mais óbvia, sem necessariamente ter agravado.

A maioria dos casos de ptose congênita não ocorre devido a fatores genéticos ${ }^{(14)}$, mas existe uma relação com herança autossômica dominante ${ }^{(5)}$. Foi encontrado neste estudo uma baixa prevalência de antecedentes familiares de ptose $(11,9 \%)$. Em outros estudos, esta prevalência foi de $7,9 \%^{(12)}$ e $32,3 \%{ }^{(15)}$. Por conter uma amostra pequena, este estudo encontrou apenas um caso de blefarofimose e, deste modo, não foi possível verificar se houve concordância com a literatura, a qual relata que uma história familiar de ptose positiva é mais prevalente nos casos de síndromes genéticas como a blefarofimose ${ }^{(5,6)}$.

Ao se falar da lateralidade, este estudo se aproxima dos dados da literatura, a qual informa que a unilateralidade ocorre, em média, em $70 \%$ dos $\operatorname{casos}^{(5,14)}$. Outros autores encontraram prevalência de unilateralidade ainda mais alta, variando de $76,2 \%$ a $83 \%^{(11,13,16)}$.

Quando a ptose obstrui parcialmente o campo visual, o paciente tende a elevar o mento para poder enxergar o horizonte, posição esta conhecida como "mento elevado" ou "chin up". Neste estudo foi encontrada uma maior prevalência dessa situação nos casos em que a ptose era bilateral.
O estrabismo e a obstrução visual causada pela ptose estão entre os principais fatores associados à ambliopia ${ }^{(8,9)}$. Reconhecer precocemente os fatores de risco ambliogênicos pode aumentar a chance de recuperação da acuidade visual por meio de tratamento cirúrgico precoce ${ }^{(7-9)}$. Neste estudo foi encontrado uma prevalência de $17 \%$ de ambliopia, não ficando distante dos $21,5 \%$ encontrados em um estudo mais recente ${ }^{(11)}$, embora seja superior ao encontrado em outros estudos mais antigos $\left(5,38 \%^{(12)}\right.$ e $\left.5,9 \%^{(13)}\right)$. Quanto ao estrabismo, este esteve associado às ptoses congênitas em 19\% dos casos, um valor que está próximo aos encontrados em outros estudos $\left(16,2 \%{ }^{(11)}\right.$ e $\left.25 \%{ }^{(16)}\right)$. Embora seja uma amostra pequena, foi encontrado neste estudo, com fins descritivos, uma prevalência de estrabismo e ambliopia maior que a da população geral, na qual é possível encontrar estrabismo em torno de $2 \%$ e ambliopia em torno de $5 \%{ }^{(8)}$. A literatura relata que a maior prevalência de ambliopia nos portadores de ptose congênita deve ser devido a maior prevalência de fatores ambliogênicos encontrados nestes pacientes ${ }^{(11)}$.

O fenômeno de Marcus Gunn é descrito como sendo responsável por 2 a $13 \%$ das ptoses congênitas ${ }^{(2)}$. Neste trabalho, este fenômeno foi encontrado em 9,5\% dos casos e, em outros trabalhos epidemiológicos, foram descritos $1,2 \%^{(12)}$ e 5,3\%.(13) A síndrome da blefarofimose foi encontrada em apenas um caso, sendo que em um estudo realizado em São Paulo foi encontrado um valor de $6,3 \%^{(13)}$. O glaucoma é mais comumente encontrado como um fator associado a ptoses adquiridas, onde já foi descrita uma associação de $38,7 \%{ }^{(15)}$.

A ausência de prega palpebral é um achado característico das ptoses congênitas ${ }^{(5,6,14)}$. Neste estudo, pouco mais da metade dos olhos ptóticos $(52,5 \%)$ não possuía esta prega.

A excursão do MEPS foi encontrada como fraca ou ausente em $63 \%$ dos olhos, e a classificação da ptose como severa foi a mais prevalente $(38,5 \%)$. Analisando-se com fins descritivos, $71,4 \%$ das ptoses severas tinham excursão do MEPS fraca, sendo que em outro estudo esta associação foi encontrada em 78,3\% ${ }^{(15)}$. Esses resultados entram em acordo com a literatura, que mostra uma maior prevalência destes achados nas ptoses congênitas ${ }^{(3,4)}$.

A suspensão Frontal é um dos procedimentos cirúrgicos mais utilizados para ptoses severas com excursão do MEPS pobre ou ausente, e também pode ser utilizada para blefarofimose ou fenômeno de Marcus Gun, sendo, portanto, muito utilizada para ptoses congênitas ${ }^{(3,4)}$. Concordando com a literatura, a correção cirúrgica por 
elevação ao Frontal foi a mais indicada, abrangendo $75 \%$ dos casos cirúrgicos. A excursão do MEPS normal ou boa, que é mais prevalente nas ptoses adquiridas, foi encontrada em uma menor percentagem (19,5\%).

Embora o tratamento para as ptoses seja cirúrgi$\mathrm{co}^{(4)}$, pode-se prorrogar a cirurgia quando esta não for urgente, a fim de se obter mensurações mais acuradas em uma criança mais colaborativa ${ }^{(5)}$. Tiveram indicação cirúrgica $57,2 \%$ dos casos deste estudo, sendo que em outros trabalhos foram encontrados $38,5 \%^{(11)} \mathrm{e}$ $63,6 \%{ }^{(15)}$. A mediana da idade dos pacientes cirúrgicos foi de 7,5 anos e, em outro estudo, a média foi de 4,6 $\operatorname{anos}^{(11)}$.

Por problemas de arquivamento dos prontuários no hospital em estudo, a amostra encontrada acabou sendo menor que a esperada, tornando-se este um fator limitante deste trabalho, além do delineamento transversal.

A visão é um dos cinco sentidos por meio dos quais percebemos o mundo, e todos os esforços devem ser feitos em prol desta. Como a ptose congênita pode levar a ambliopia ${ }^{(7-9)}$, os avanços da medicina oftalmológica, desde a avaliação inicial do paciente até a indicação da melhor técnica cirúrgica, são de extrema importância para que haja resultados terapêuticos eficazes para esta condição. No presente estudo, embora descritivo e com uma amostra pequena, a prevalência de ambliopia nestes pacientes foi maior que a da população geral, reforçando a importância de uma avaliação precoce dos pacientes com ptose congênita.

\section{ReFERÊNCIAS}

1. Cruz AAV, Chahud F, Guimarães FC. Patologia dos anexos oculares. Medicina (Ribeirão Preto). 1997;30(1):36-51.

2. Torres MRF, Calixto Júnior N, Oliveira LR, Steiner SA, Iscold AM. Fenômeno de Marcus Gunn: diagnóstico diferencial das ptoses palpebrais na criança. J Pediatr (Rio J). 2004;80(3):249-52.

3. Baroody M, Holds JB, Vick VL. Advances in the diagnosis and treatment of ptosis. Curr Opin Ophthalmol. 2005;16(6):351-5.

4. Cetinkaya A, Brannan PA. Ptosis repair options and algo- rithm. Curr Opin Ophthalmol. 2008;19(5):428-34.

5. Suh DW. Ptosis, congenital [Internet]. [cited 2009 May 6]. Available from: <http://www.emedicine.com/oph/ TOPIC345.HTM.> Acessado em: 06 mai 2009.

6. Figueiredo ARP, Souza GL, Almeida HC, Oliveira LRM, Magalhães MM, Portellinha WM. Blefaroptose. In: Soares EJC, Moura EM, Gonçalves JOR. Cirurgia plástica ocular. São Paulo: Roca; 2002. p.77-152

7. Wensveen JM, Harwerth RS, Hung L, Ramamirtham R, Kee CS, Smith EL 3rd. Brief daily periods of unrestricted vision can prevent form-deprivation amblyopia. Invest Ophthalmol Vis Sci. 2006;47(6):2468-77.

8. Adams GG, Sloper JJ. Update on squint and amblyopia. J R Soc Med. 2003;96(1):3-6. Review.

9. Doshi NR, Rodriguez ML. Amblyopia. Am Fam Physician. 2007;75(3):361-7. Review.

10. Paixão MP, Miot HA, Machado Filho CDS. Avaliação do impacto da blefaroplastia superior na qualidade de vida utilizando questionário padronizado (Qblefaro): estudo piloto. An Bras Dermatol. 2008;83(1):32-7.

11. Lin LK, Uzcategui N, Chang EL. Effect of surgical correction of congenital ptosis on amblyopia. Ophthal Plast Reconstr Surg. 2008;24(6):434-6.

12. González S E, Gutiérrez C LF. Ptosis palpebral en la Fundacion Oftalmologica Colombiana: estudio retrospectivo de 148 casos. Medellin 1984-1988. CES Med. 1989;3(1):19-23.

13. Lucci LMD, Portellinha W, Sant'Ana AEBPP. Ptose palpebral: estudo de 390 casos. Arq Bras Oftalmol. 1997;60(5):455-7.

14. Skorin L Jr. Blepharoptosis and its management [Internet]. [cited 2009 May 6]. Available from:

ht tp://www.optometry.myzen.co.uk/articles/docs/ a5039c1bb8168ff7a0b813cbb463b6bc_skorin20011102.pdf

15. Herrera Soto M, González Rodríguez C, Martínez Suárez N, Padilla González C. Comportamiento de la ptosis palpebral en el servicio de oculoplastia. Año 2001. Rev Cuba Oftalmol. 2002;15(2).

16. Della Paollera M. Coincidência de blefaroptose com outras alterações oftalmológicas. Arq Bras Oftalmol. 1988;51(2):94-5.

\author{
Endereço para correspondência: \\ Hospital Regional Homero de Miranda Gomes, \\ Secretaria Estadual de Saúde \\ Rua Adolfo Donato da Silva, s/n \\ Praia Comprida \\ CEP 88103-901 - São José (SC), Brasil \\ Tel: (48)3271-9280 \\ e-mail: astorg@uol.com.br
}

